\title{
Metabolic Syndrome and Related Factors in Chinese Children and Adolescents: Analysis from a Chinese National Study
}

\author{
Yanna Zhu ${ }^{1}$, Hao Zheng ${ }^{1}$, Zhiyong Zou ${ }^{2}$, Jin Jing ${ }^{1}$, Yinghua $\mathrm{Ma}^{2}$, Haijun Wang ${ }^{2}$, Jiayou Luo ${ }^{3}$, Xin Zhang ${ }^{4}$, \\ Chunyan Luo ${ }^{5}$, Hong Wang ${ }^{6}$, Haiping Zhao ${ }^{7}$, Dehong Pan ${ }^{8}$, Per Sangild ${ }^{9}$, Biraj M Karmacharya ${ }^{10}$, Jun Ma ${ }^{2}$ \\ and Yajun Chen ${ }^{1}$
}

Yanna Zhu and Hao Zheng contributed equally to this work.

Yajun Chen and Jun Ma are joint senior authors.

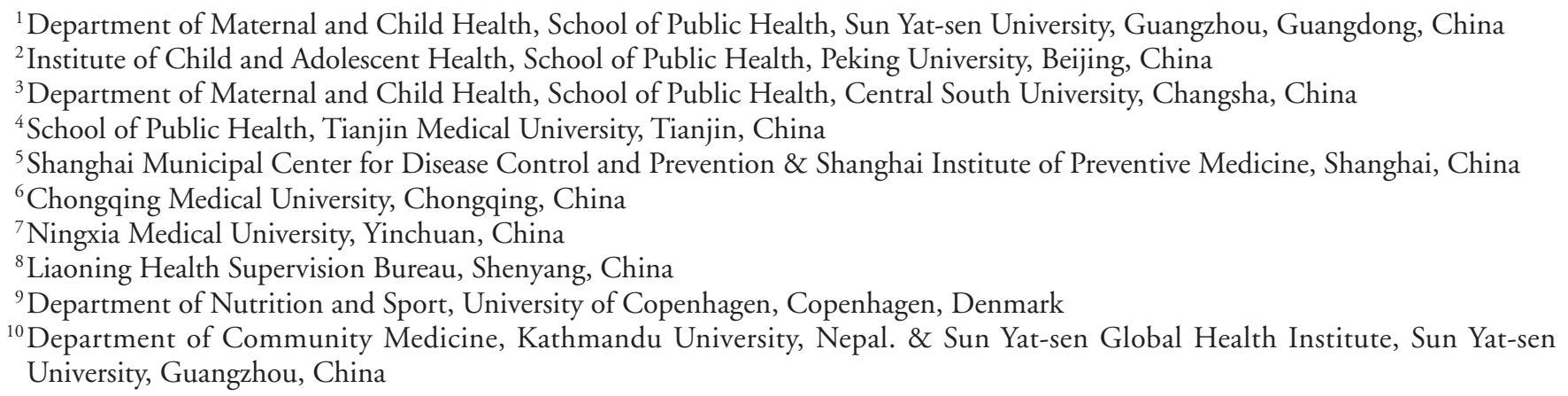

Aims: Metabolic syndrome (MetS) has become a worldwide epidemic; however, few studies have described its status in Chinese children. This study aimed to estimate MetS status and its associations with geography, economic development, birth weight, and parental education of Chinese children and adolescents.

Methods: Data were derived from 15,045 participants aged 7-18 years across seven Chinese provinces. Physical measurement and blood tests were conducted to assess the five classical MetS components described by the International Diabetes Federation, including abdominal obesity (the essential component), high blood pressure, low high-density cholesterol (HDL-C), high triglyceride, and elevated fasting glucose (FG). Logistic regression was adopted to explore possible associations between MetS and other factors.

Results: Overall, MetS prevalence was $2.3 \%$, higher in males $(2.8 \%$ vs. $1.7 \%$ in females), northern regions $(3.1 \%)$, more developed regions (2.9\%), and older participants (aged $16-18$ years) $(P<0.05$ for all). Among the five MetS components, abdominal obesity and low HDL-C level were most prevalent $(21.8 \%$ and $14.4 \%)$, and $35.9 \%$ of the participants had at least one component. In logistic regression, MetS itself did not correlate with birth weight or parental education. High birth weight was positively correlated with abdominal obesity (odds ratio, 1.48 ) butnegatively associated with elevated FG (odds ratio, 0.49 ).

Concusions: MetS itself was not common in Chinese children and adolescents, whereas its certain components were far more prevalent. Children from North China, more-developed areas, and at an older age were more likely to develop MetS. Strategies designed to prevent pediatric MetS in China should focus on prevalent components as well as its geographic and economic development predilections.

Key words: Metabolic syndrome, Abdominal obesity, Children and adolescents

\section{Introduction}

Metabolic syndrome (MetS) is defined by a clus- ter of a series of factors associated with metabolic cardiovascular diseases and was first described by Kylin in the $1920 \mathrm{~s}^{1)}$. Previous studies showed that the syn- 
drome was closely associated with an increased risk of developing type 2 diabetes and cardiovascular diseases $(\mathrm{CVDs})^{2)}$. Prospective studies in children and adolescents have also revealed that MetS in childhood is associated with MetS and type 2 diabetes in adulthood $^{3)}$. Therefore, early diagnosis and preventive interventions of MetS are becoming increasingly important for improving health outcomes during adolescence and reducing the incidence of CVDs in adults. Because there is no accepted standard for diagnosis of pediatric MetS yet, it remains difficult to compare the prevalence in children and adolescents among different countries.

Abdominal obesity, high blood pressure (BP), low high-density cholesterol (HDL-C) level, high triglyceride (TG), and elevated fasting glucose (FG) are included in all existing MetS definitions ${ }^{1,4-6)}$; however, different cutoff levels may lead to widely different estimates, ranging from $0 \%-50 \%$ in various populations $^{7-10)}$. Therefore, it is difficult to estimate the global epidemic of pediatric MetS and make comparisons among countries and regions. To diagnose the syndrome in children and adolescents, the International Diabetes Federation (IDF) had proposed a definition for those aged 10-18 years, in 2007 ${ }^{6}$. According to the IDF definition, MetS diagnosis requires the presence of abdominal obesity (waist circumference, WC $\geq 90$ th percentile) and any two of the following four factors: 1) serum TG $\geq 1.7 \mathrm{mmol} / \mathrm{L}, 2) \mathrm{HDL}$ cholesterol $<1.03 \mathrm{mmol} / \mathrm{L}, 3)$ systolic $\mathrm{BP} \geq 130$ $\mathrm{mmHg}$ and/or diastolic $\mathrm{BP} \geq 85 \mathrm{mmHg}$, and 4) FG $\geq$ $5.6 \mathrm{mmol} / \mathrm{L}$ or presence of diabetes mellitus. Unlike other definitions, the IDF definition considers abdominal obesity as a precondition, thereby making the definition more applicable for Chinese population, because studies among Asian population have revealed that large WC is closely associated with diabetes and CVDs compared with other MetS components ${ }^{11,12)}$. This ethnic predilection may result from a relatively low body mass index (BMI) and more body fat in Asians ${ }^{13)}$. Therefore, to diagnose MetS in China, the above IDF definition should be considered, using age-, sex-, and ethnicity-specific cutoff points (of WC) for Chinese children and adolescents ${ }^{14)}$.

MetS in childhood may predispose to MetS and CVDs in adulthood; therefore, it is important to identify the MetS risk factors in childhood. Researchers worldwide have proposed different factors that might contribute to the development of MetS. Birth weight, parental education, age, gender, and ethnicity are among the factors suggested to affect MetS by previous studies ${ }^{15}$. Low birth weight (LBW) contributes to MetS development in adulthood ${ }^{16)}$, but the relation remains unclear between children and adolescents. Some studies suggested that high parental education protects children against pediatric MetS, but the results were not consistent ${ }^{17)}$

Results from studies on pediatric MetS in China are inconsistent, possibly because of small sample sizes, short age span, or regional factors. The objective of this study was to estimate the status of IDF-defined MetS and its five components in a nationwide crosssectional sample of Chinese children and adolescents aged 7-18 years. Further, we attempted to clarify the relationship between MetS components and demographics, regional determinants, birth weight, and parental education, and to provide a basis for preventive interventions against pediatric MetS in China.

\section{Methods}

\section{Study Sample}

This study was conducted as a part of the baseline survey of an interventional project, designed as a nationwide, multi-centered, and cluster-randomized controlled trial involving $>70,000$ participants from seven provinces of China (Guangzhou, Chongqing, Hunan, Tianjin, Shanghai, Liaoning, and Ningxia). Here, it was tested whether the so called "the health lifestyles interventions" would help to prevent obesity in Chinese children and adolescents. The detailed sampling and recruitment methods and data collection procedures were described previously ${ }^{18)}$. The participants in the present study represent a subsample of the original intervention project. Informed written consent or assent was obtained from both children and their parents. The study protocol was approved by the Ethical Committee of Peking University.

\section{Justification of Sample Size}

The formula for estimating a sample size in a single cross-sectional survey was adopted to calculate the sample size. We used the MetS prevalence (1.4\%) based on a study among 22,071 children and adolescents across 6 cities of China (2013), to calculate the sample size. It came to 8,419 and increased to 10,524 after considering $80 \%$ power.

In the present study, data were analyzed for

Address for correspondence: Yajun Chen, Mailing address: Department of Maternal and Child Health, School of Public Health, Sun Yat-sen University, No.74 Zhongshan Road II,Guangzhou, PR-China E-mail: chenyj68@sysu.edu.cn.

Received: April 28, 2019 Accepted for publication: September 5, 2019

Copyright@2020 Japan Atherosclerosis Society

This article is distributed under the terms of the latest version of CC BY-NC-SA defined by the Creative Commons Attribution License. 
16,540 participants who had complete data for all five MetS components. Participants were excluded if they were younger than 7 or older than 18 years $(n=989)$ or did not have BMI value $(n=506)$. Thus, 1,495 participants did not meet the inclusion criteria, resulting in a final sample size of 15,045 participants $(7,711$ males and 7,334 females) aged 7-18 years. The final sample size $(15,045)$ was larger than the result of sample size calculation $(10,524)$ and qualified for detecting the differences in MetS prevalence among groups.

\section{Anthropology}

The anthropometric data in our study were measured by trained nurses, according to standardized methods, with the participant wearing light clothing. Height was measured to the nearest $0.1 \mathrm{~cm}$ with a stadiometer; participants were standing shoeless in an upright position. Body weight was measured to the nearest $0.1 \mathrm{~kg}$ using a self-zeroing scale; participants were standing shoeless in standing position and having empty pockets. WC was measured to the nearest $0.1 \mathrm{~cm}$ at $1 \mathrm{~cm}$ above the umbilicus with a nonelastic tape, with the participant in a standing position. In the present study, WC $\geq$ the 90th percentile for age and sex was defined as abdominal obesity, according to the cutoff values published by the Chinese National Institute of Nutrition and Food Safety ${ }^{14)}$. Diastolic and systolic blood pressure (DBP and SBP) measurements were obtained from the right arm. After resting quietly in a sitting position for $5 \mathrm{~min}$, up to four consecutive BP readings were obtained with a validated mercury sphygmomanometer (model XJ1ID, China) and TZ-1 stethophone (Yuyue, China). Mean values of replicates of systolic and diastolic measurements provided estimates of current BP levels. A systolic BP mean $\geq 130 \mathrm{mmHg}$ or a diastolic $\mathrm{BP}$ mean $\geq 85$ $\mathrm{mmHg}$ was defined as high BP.

\section{Laboratory Assays}

Participants fasted for $12 \mathrm{~h}$ before the blood sampling. Any participant who did not meet the requirement was excluded from the specific blood sampling. Venous blood samples were obtained from the antecubital vein and collected into vacuum tubes before breakfast by trained nurses. Samples were centrifuged at $3000 \mathrm{r}$, aliquoted, and stored at $-80^{\circ} \mathrm{C}$. All biochemical analyses were carried out at a biomedical analyses company, accredited by Peking University. Glucose oxidase and enzymatic methods were adopted to measure FG and TG, respectively; HDL-C was measured using clearance method.

\section{Questionnaire}

To obtain information about demographic infor- mation, birth weight, and parental education, questionnaires for both children and parents were provided. Questionnaires were developed according to the so called "information, motivation, and behavior skills model." All parent-reported questionnaires were sent through their children. For children in grades 1-3, child-reported questionnaires were also completed by their parents; for other participants, childreported questionnaires were filled by themselves while in class at school, under the instruction of trained teachers. Training and a standardized operation manual were provided for the examiner, prior to the examination. Appropriate help and guidance were available to the students when filling the questionnaires, if necessary.

Data about demographics, birth weight, and parental education were obtained. The Qinling Mountain-Huaihe River line was used to divide the study area into southern and northern parts ${ }^{19)}$. Liaoning, Ningxia, and Tianjin were defined as "North China," whereas Hunan, Chongqing, Shanghai, and Guangzhou as "South China." Similarly, we classified the seven regions into more developed areas (Tianjin, Liaoning, Shanghai, and Guangzhou) and underdeveloped areas (Hunan, Chongqing, and Ningxia), according to the per capita income of the seven regions in $2016^{20)}$. Birth weight was classified into LBW, normal birth weight (NBW), and high birth weight (HBW), according to WHO guidelines in $2016^{21}$. Information about parental education was classified into none/ primary, secondary, and university/above in the present study.

\section{Definitions}

In this study, MetS was defined according to the IDF definition by the following criteria ${ }^{6}$ : abdominal obesity (WC $\geq 90$ th percentile, determined by the cutoff points for Chinese children and adolescents) ${ }^{14)}$, and the presence of two or more of the following four components: 1) serum TG $\geq 1.7 \mathrm{mmol} / \mathrm{L}, 2) \mathrm{HDL}$ cholesterol $<1.03 \mathrm{mmol} / \mathrm{L}, 3)$ systolic $\mathrm{BP} \geq 130$ $\mathrm{mmHg}$ and/or diastolic $\mathrm{BP} \geq 85 \mathrm{mmHg}$, and 4) FG $\geq$ $5.6 \mathrm{mmol} / \mathrm{L}$ or presence of diabetes mellitus. It is suggested by the IDF that MetS cannot be diagnosed among those younger than 10 years, but measurements of the five components should be made because they can be predictors for adolescent and adult metabolic syndrome. In this study, children younger than 10 years were also subjected to the IDF definition mentioned above to detect MetS and its related factors.

To investigate the weight status of the participants, obesity was defined as body mass index (BMI) for age $>$ two standard deviations above the WHO 
Table 1. Basic characters of participants

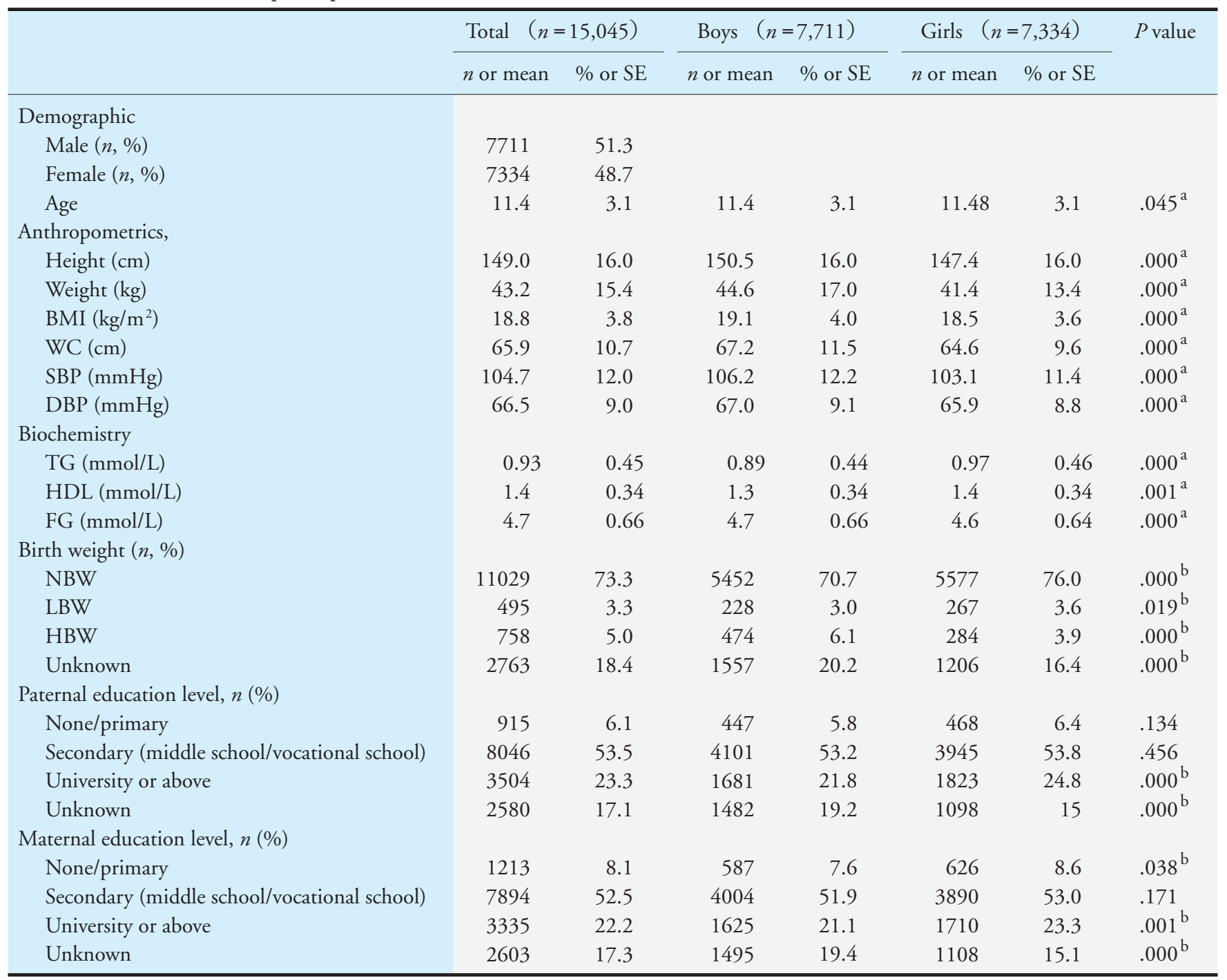

Data are number $(n)$ and percentage $(\%)$, or mean \pm standard error (SE), unless otherwise indicated.

BMI, body mass index; WC, waist circumference; SBP, systolic blood pressure; DBP, diastolic blood pressure; TG, triglyceride; HDL-C, high density cholesterol; FG, fasting glucose; NBW, normal birth weight; LBW, low birth weight, HBW, high birth weight.

a $p<0.05$, boys vs. girls, assessed by one-way ANOVA.

$\mathrm{b} p<0.05$, boys vs. girls, assessed by $\chi^{2}$ test for categorical variables.

Growth Reference median ${ }^{22)}$.

\section{Statistical Analysis}

All data were input with EpiData 3.0 software (The EpiData Association, Odense, Denmark), by double entry and validation. All analyses were performed using procedures for sample survey data that are readily available in SPSS, version 21.0. Group differences were analyzed using one-way ANOVA for continuous variables and $\chi^{2}$ test for categorical values. A one-way ANOVA was used to compare the participants by their number of MetS components. Associations between MetS prevalence and risk factors were determined using logistic regression models, after adjustments. Two-tailed $P$ values $<0.05$ were considered as statistical significant.

\section{Results}

\section{Basic Characteristics}

Demographic characteristics and clinical characteristics of the study sample are shown in Table 1, including sex, age, anthropometrics, blood lipids and glucose, birth weight, and parental education level. There were 15,045 participants $(7,711$ males and 7,334 females, i.e., $51.3 \%$ males) in the study, with an 
Table 2. Prevalence of MetS according to age, sex, geographic location and economic development level(\%)

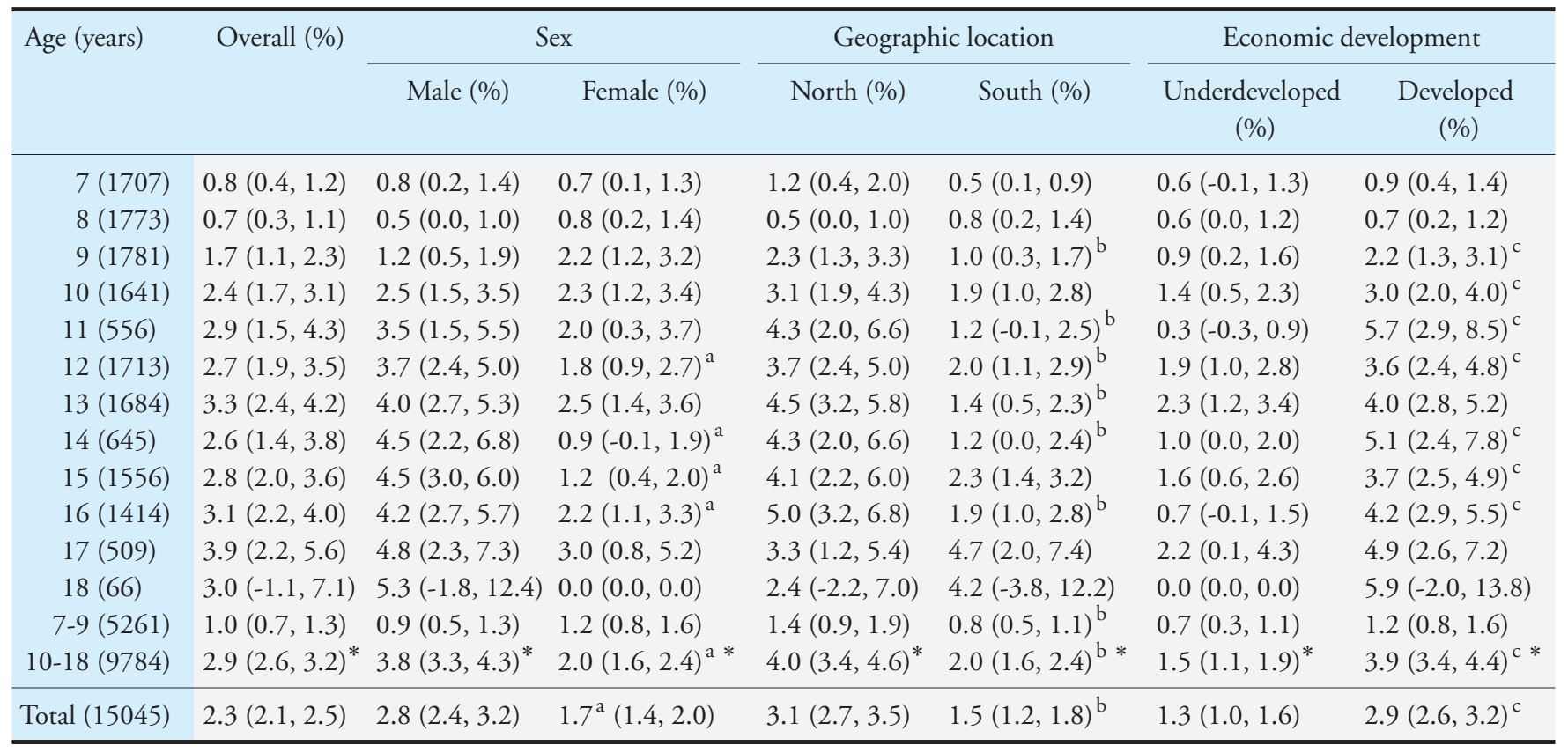

Data are percentage (\%) with $95 \%$ confidence interval. MetS, metabolic syndrome.

${ }_{0}^{a} p<0.05$, male vs. female, assessed by $\chi^{2}$ test for categorical variables.

b $p<0.05$, north vs. south, assessed by $\chi^{2}$ test for categorical variables.

${ }_{*} p<0.05$, underdeveloped vs. developed, assessed by $\chi^{2}$ test for categorical variables,

$* p<0.05,7-9$ vs. $10-18$.

average age of 11.4 years. Gender differences were found in almost all the variables mentioned in Table 1 , except for part of the parental education.

Overall, the prevalence of obesity (defined by BMI for age) was $9.2 \%$ in Chinese children and adolescents, higher in males (13.3\% vs. $4.8 \%$ in females), northern regions ( $11.3 \%$ vs. $7.4 \%$ in the south), more developed regions $(11.7 \%$ vs. $5.4 \%$ in underdeveloped regions), and in younger participants $(11.9 \%$ in children aged $7-9$ years vs. $7.7 \%$ in children aged $16-18$ years) $(P<0.05$ for all $)$.

\section{Prevalence of MetS}

Prevalence of MetS assessed by age, sex, geographic location and economic development level is shown in Table 2. The overall MetS prevalence was $2.3 \%$, higher in males than in females $(2.8 \%$ vs. $1.7 \%$, $P<0.05)$, in North China than in South China $(3.1 \%$ vs. $1.5 \%)$, and in more developed areas than in underdeveloped areas $(2.9 \%$ vs. $1.3 \%)(P<0.05$ for all). Furthermore, the MetS prevalence was higher in older participants (16-18 years vs. 10-15 years), peaked at the age of 17 years $(3.9 \%)$, but slightly dropped to $3.0 \%$ at the age of 18 years.

\section{Prevalence of Individual MetS Components}

Distribution of individual MetS components is presented in Table 3. Overall, abdominal obesity and low HDL-C level were most prevalent $(21.8 \%$ and $14.4 \%$, respectively), whereas elevated FG was the least common component $(3.0 \%)$. The prevalence of abdominal obesity was higher in the north and more developed areas of China $(24.2 \%$ and $25.9 \%$, respectively, both $P<0.05)$. No significant gender differences in abdominal obesity were observed. Males had higher rates of high $\mathrm{BP}$ ( $4.6 \%$ vs. $2.7 \%$ ), low HDL cholesterol level (15.8\% vs. $12.9 \%)$, and elevated FG $(4.1 \%$ vs. $1.8 \%)$ but with lower rates of high TG (5.0\% vs. $6.0 \%)$, compared with their female counterparts (all $P<0.05$ ). Geographic differences were found in all five components with all being more prevalent in North China $(\mathrm{P} \leq 0.05)$.

Prevalence of individual MetS components according to age is shown in Fig.1 (A-E). Among the five components, abdominal obesity was prevalent in all age groups, whereas the other four components were generally more prevalent in older participants (aged 10-18 years) than younger ones (aged 7-9 years).

\section{Prevalence of MetS Components Combinations}

Prevalence of participants with one or more components of MetS is presented in Table 4. Among those participants, $64.1 \%$ had no component, $26.0 \%$ had 
Table 3. Prevalence of individual components of MetS among 15045 Chinese children and adolescents aged 7-18 years, 2013

\begin{tabular}{|c|c|c|c|c|c|c|c|c|c|c|c|}
\hline \multirow[b]{2}{*}{ Component } & \multirow[b]{2}{*}{$n$} & \multirow[b]{2}{*}{$\begin{array}{c}\text { Overall } \\
(n=15,045)\end{array}$} & \multicolumn{3}{|c|}{ Sex } & \multicolumn{3}{|c|}{ Geographic location } & \multicolumn{3}{|c|}{ Economical development } \\
\hline & & & $\begin{array}{c}\text { Male } \\
(n=7,711)\end{array}$ & $\begin{array}{c}\text { Female } \\
(n=7,334)\end{array}$ & $P$ & $\begin{array}{c}\text { North } \\
(n=6,843)\end{array}$ & $\begin{array}{c}\text { South } \\
(n=8,202)\end{array}$ & $P$ & $\begin{array}{c}\text { Under } \\
\text { developed } \\
(n=5,997)\end{array}$ & $\begin{array}{l}\text { Developed } \\
(n=9,048)\end{array}$ & $P$ \\
\hline $\begin{array}{l}\text { Abdominal } \\
\text { obesity }\end{array}$ & 3282 & $\begin{array}{c}21.8 \\
(21.1,22.5)\end{array}$ & $\begin{array}{c}21.2 \\
(20.3,22.1)\end{array}$ & $\begin{array}{c}22.5 \\
(21.5,23.5)\end{array}$ & 0.06 & $\begin{array}{c}24.2 \\
(23.2,25.2)\end{array}$ & $\begin{array}{c}19.8 \\
(18.9,20.7)\end{array}$ & $0.00^{\mathrm{b}}$ & $\begin{array}{c}15.7 \\
(14.8,16.6)\end{array}$ & $\begin{array}{c}25.9 \\
(25.0,26.8)\end{array}$ & $0.00^{\mathrm{c}}$ \\
\hline High BP & 559 & $\begin{array}{c}3.7 \\
(3.4,4.0)\end{array}$ & $\begin{array}{c}4.6 \\
(4.1,5.1)\end{array}$ & $\begin{array}{c}2.7 \\
(2.3,3.1)\end{array}$ & $0.00^{\mathrm{a}}$ & $\begin{array}{c}6.4 \\
(5.8,7.0)\end{array}$ & $\begin{array}{c}1.5 \\
(1.2,1.8)\end{array}$ & $0.00^{\mathrm{b}}$ & $\begin{array}{c}4.1 \\
(3.6,4.6)\end{array}$ & $\begin{array}{c}3.5 \\
(3.1,3.9)\end{array}$ & 0.08 \\
\hline Low HDL-C & 2163 & $\begin{array}{c}14.4 \\
(13.8,15.0)\end{array}$ & $\begin{array}{c}15.8 \\
(15.0,16.6)\end{array}$ & $\begin{array}{c}12.9 \\
(12.1,13.7)\end{array}$ & $0.00^{\mathrm{a}}$ & $\begin{array}{c}19.0 \\
(18.1,19.9)\end{array}$ & $\begin{array}{c}10.5 \\
(9.8,11.2)\end{array}$ & $0.00^{\mathrm{b}}$ & $\begin{array}{c}15.5 \\
(14.6,16.4)\end{array}$ & $\begin{array}{c}13.6 \\
(12.9,14.3)\end{array}$ & $0.00^{\mathrm{c}}$ \\
\hline High TG & 826 & $\begin{array}{c}5.5 \\
(5.1,5.9)\end{array}$ & $\begin{array}{c}5.0 \\
(4.5,5.5)\end{array}$ & $\begin{array}{c}6.0 \\
(5.5,6.5)\end{array}$ & $0.01^{\mathrm{a}}$ & $\begin{array}{c}6.1 \\
(5.5,6.7)\end{array}$ & $\begin{array}{c}5.0 \\
(4.5,5.5)\end{array}$ & $0.00^{\mathrm{b}}$ & $\begin{array}{c}5.2 \\
(4.6,5.8)\end{array}$ & $\begin{array}{c}5.7 \\
(5.2,6.2)\end{array}$ & 0.27 \\
\hline Elevated FG & 454 & $\begin{array}{c}3.0 \\
(2.7,3.3)\end{array}$ & $\begin{array}{c}4.1 \\
(3.7,4.5)\end{array}$ & $\begin{array}{c}1.8 \\
(1.5,2.1)\end{array}$ & $0.00^{\mathrm{a}}$ & $\begin{array}{c}4.1 \\
(3.6,4.6)\end{array}$ & $\begin{array}{c}2.1 \\
(1.8,2.4)\end{array}$ & $0.00^{\mathrm{b}}$ & $\begin{array}{c}0.7 \\
(0.5,0.9)\end{array}$ & $\begin{array}{c}4.6 \\
(4.2,5.0)\end{array}$ & $0.00^{\circ}$ \\
\hline
\end{tabular}

Data are percentage with $95 \%$ confidence interval.

MetS, metabolic syndrome; BP, blood pressure; HDL-C, HDL cholesterol; TG, triglyceride; FG, fasting glucose.

${ }_{p}<0.05$, male vs. female, assessed by $\chi^{2}$ test for categorical variables.

b $p<0.05$, north vs. south, assessed by $\chi^{2}$ test for categorical variables.

${ }^{c} p<0.05$, underdeveloped vs. developed, assessed by $\chi^{2}$ test for categorical variables.

Fig.1A Prevalence of abdominal obesity.

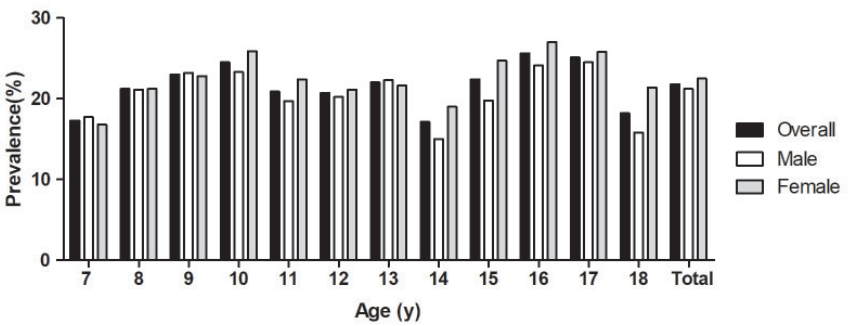

Fig.1C Prevalence of low HDL-C level.

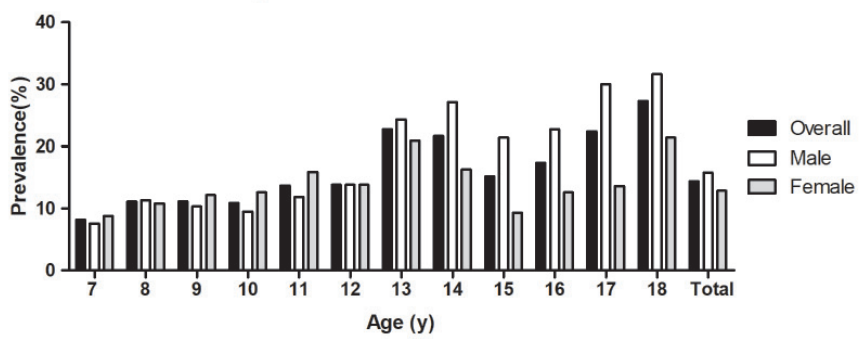

Fig.1E Prevalence of elevated fasting glucose.

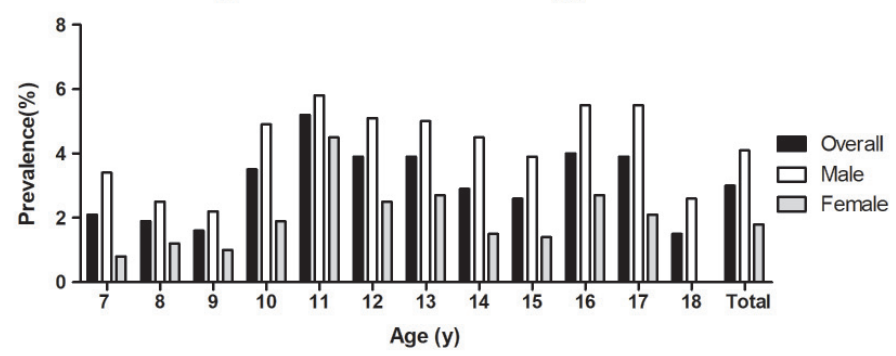

Fig.1B Prevalence of high blood pressure.

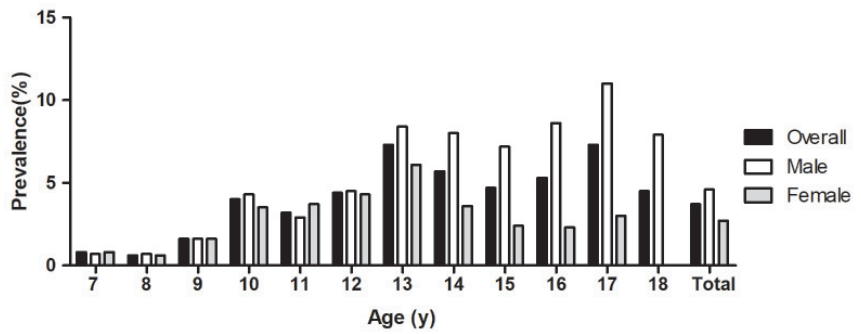

Fig.1D Prevalence of high triglyceride level.

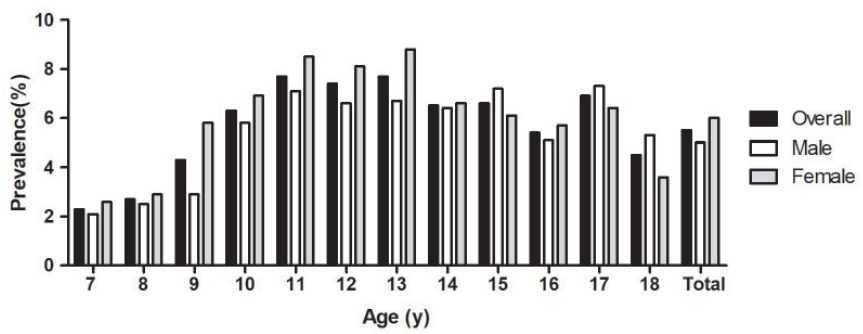

Fig. 1. Prevalence of individual components of MetS by age among Chinese children and adolescents, 2013

Notes: Data are in percentage

Abbreviations: MetS, metabolic syndrome; abdominal obesity, WC

$\geq$ P90; high blood pressure, SBP $\geq 130 \mathrm{mmHg}$ or $\mathrm{DBP} \geq 85$ $\mathrm{mmHg}$; low HDL-C level, HDL-C $<1.03 \mathrm{mmol} / \mathrm{L}$; high triglyceride level, triglyceride $(\mathrm{TG}) \geq 1.7 \mathrm{mmol} / \mathrm{L}$; elevated fasting glucose $(\mathrm{FG}), \mathrm{FG} \geq 5.6 \mathrm{mmol} / \mathrm{L}$. 
Table 4. Prevalence of one or more components of MetS among 15045 Chinese children and adolescents, 2013

\begin{tabular}{|c|c|c|c|c|c|c|c|c|c|c|c|c|c|c|c|}
\hline & \multicolumn{15}{|c|}{ Number of MetS components } \\
\hline & \multicolumn{3}{|c|}{ Abdominal obesity only } & \multicolumn{3}{|c|}{ Abdominal obesity +1} & \multicolumn{3}{|c|}{ Abdominal obesity +2} & \multicolumn{3}{|c|}{ Abdominal obesity +3} & \multicolumn{3}{|c|}{ Abdominal obesity +4} \\
\hline & Overall & Male & Female & Overall & Male & Female & Overall & Male & Female & Overall & Male & Female & Overall & Male & Female \\
\hline \multicolumn{16}{|l|}{ Age (yr) } \\
\hline 7 (1707) & 13.9 & 14.3 & 13.6 & 2.6 & 2.6 & 2.6 & 0.7 & 0.7 & 0.7 & 0.1 & 0.1 & 0.0 & 0.0 & 0.0 & 0.0 \\
\hline $8(1773)$ & 16.5 & 16.7 & 16.3 & 4.0 & 3.9 & 4.1 & 0.7 & 0.5 & 0.8 & 0.0 & 0.0 & 0.0 & 0.0 & 0.0 & 0.0 \\
\hline $9(1781)$ & 15.9 & 16.3 & 15.6 & 5.4 & 5.7 & 5.0 & 1.6 & 1.1 & 2.1 & 0.1 & 0.1 & 0.1 & 0.0 & 0.0 & 0.0 \\
\hline $10(1641)$ & 15.4 & 14.7 & 16.1 & 6.7 & 6.1 & 7.5 & 2.1 & 2.1 & 2.2 & 0.3 & 0.4 & 0.1 & 0.0 & 0.0 & 0.0 \\
\hline $11(556)$ & 12.1 & 9.4 & $15.4^{\mathrm{a}}$ & 5.9 & 6.8 & 4.9 & 2.7 & 3.2 & 2.0 & 0.0 & 0.0 & 0.0 & 0.2 & 0.3 & 0.0 \\
\hline $12(1713)$ & 11.6 & 11.0 & 12.3 & 6.3 & 5.6 & 7.0 & 2.2 & 2.7 & 1.6 & 0.5 & 0.8 & 0.2 & 0.1 & 0.1 & 0.0 \\
\hline $13(1684)$ & 11.2 & 10.6 & 11.9 & 7.5 & 7.7 & 7.2 & 2.7 & 3.1 & 2.4 & 0.5 & 0.9 & $0.1^{\mathrm{a}}$ & 0.0 & 0.0 & 0.0 \\
\hline $14(645)$ & 10.1 & 6.4 & $13.6^{\mathrm{a}}$ & 4.3 & 4.1 & 4.5 & 2.0 & 3.5 & $0.6^{\mathrm{a}}$ & 0.5 & 0.6 & 0.3 & 0.2 & 0.3 & 0.0 \\
\hline $15(1556)$ & 13.5 & 8.7 & $18.0^{\mathrm{a}}$ & 6.0 & 6.7 & 5.5 & 2.4 & 3.7 & $1.1^{\mathrm{a}}$ & 0.4 & 0.8 & $0.1^{\mathrm{a}}$ & 0.0 & 0.0 & 0.0 \\
\hline $16(1414)$ & 14.2 & 9.4 & $18.6^{\mathrm{a}}$ & 8.3 & 10.5 & $6.2^{\mathrm{a}}$ & 2.5 & 3.4 & $1.6^{\mathrm{a}}$ & 0.6 & 0.6 & 0.5 & 0.1 & 0.1 & 0.0 \\
\hline 17 (509) & 12.2 & 9.2 & $15.7^{\mathrm{a}}$ & 9.0 & 10.6 & 7.2 & 3.5 & 4.0 & 3.0 & 0.4 & 0.7 & 0.0 & 0.0 & 0.0 & 0.0 \\
\hline $18(66)$ & 12.1 & 5.3 & $21.4^{\mathrm{a}}$ & 3.0 & 5.3 & 0.0 & 3.0 & 5.3 & 0.0 & 0.0 & 0.0 & 0.0 & 0.0 & 0.0 & 0.0 \\
\hline Total (15045) & 13.7 & 12.3 & $15.2^{\mathrm{a}}$ & 5.8 & 6.1 & 5.5 & 1.9 & 2.3 & $1.6^{\mathrm{a}}$ & 0.3 & 0.5 & $0.1^{\mathrm{a}}$ & 0.0 & 0.1 & 0.0 \\
\hline
\end{tabular}

Data are percentage $(\%)$.

MetS, metabolic syndrome.

*Highest prevalence of a certain number of MetS components among all ages.

${ }^{a} p<0.05$, male vs. female, assessed by $\chi^{2}$ test for categorical variables.

Table 5. Multivariate logistic regression analysis of MetS and its components with birth weight and parental education $(n=11,696)$

\begin{tabular}{|c|c|c|c|c|c|c|c|c|c|c|c|c|}
\hline & \multicolumn{2}{|c|}{ MetS } & \multicolumn{2}{|c|}{ Abdominal obesity } & \multicolumn{2}{|c|}{ Low HDL-C } & \multicolumn{2}{|c|}{ Elevated FG } & \multicolumn{2}{|c|}{ High triglyceride } & \multicolumn{2}{|c|}{ High BP } \\
\hline & OR & $95 \% \mathrm{CI}$ & OR & $95 \% \mathrm{CI}$ & OR & $95 \% \mathrm{CI}$ & OR & $95 \% \mathrm{CI}$ & OR & $95 \% \mathrm{CI}$ & OR & $95 \% \mathrm{CI}$ \\
\hline \multicolumn{13}{|l|}{ Birth weight } \\
\hline ABW & 1.00 & Ref. & 1.00 & Ref. & 1.00 & Ref. & 1.00 & Ref. & 1.00 & Ref. & 1.00 & Ref. \\
\hline LBW & 0.80 & $0.39,1.63$ & 0.87 & $0.68,1.11$ & 1.21 & $0.92,1.59$ & 0.93 & $0.53,1.64$ & 0.87 & $0.55,1.36$ & 1.52 & $0.94,2.45$ \\
\hline HBW & 0.87 & $0.53,1.43$ & $1.48^{*}$ & $1.25,1.75$ & 0.98 & $0.79,1.23$ & $0.49^{*}$ & $0.28,0.84$ & 0.86 & $0.61,1.22$ & 1.31 & $0.91,1.89$ \\
\hline \multicolumn{13}{|l|}{ Paternal education } \\
\hline None/primary & 1.00 & Ref. & 1.00 & Ref. & 1.00 & Ref. & 1.00 & Ref. & 1.00 & Ref. & 1.00 & Ref. \\
\hline Secondary & 1.51 & $0.86,2.65$ & $1.30^{*}$ & $1.07,1.58$ & 0.93 & $0.75,1.44$ & 1.24 & $0.79,1.95$ & 0.90 & $0.66,1.23$ & 1.04 & $0.70,1.55$ \\
\hline University & 1.07 & $0.56,2.58$ & $1.30^{*}$ & $1.04,1.62$ & 0.81 & $0.63,1.04$ & 1.32 & $0.78,2.22$ & 0.85 & $0.58,1.23$ & 0.86 & $0.53,1.41$ \\
\hline \multicolumn{13}{|l|}{ Maternal education } \\
\hline None/primary & 1.00 & Ref. & 1.00 & Ref. & 1.00 & Ref. & 1.00 & Ref. & 1.00 & Ref. & 1.00 & Ref. \\
\hline Secondary & 1.55 & $0.95,2.53$ & 1.11 & $0.94,1.31$ & 0.99 & $0.82,1.20$ & 1.08 & $0.74,1.58$ & 1.05 & $0.79,1.40$ & 1.00 & $0.70,1.42$ \\
\hline University & 1.57 & $0.88,2.79$ & $1.35^{*}$ & $1.10,1.64$ & 0.83 & $0.65,1.05$ & 0.99 & $0.63,1.59$ & 1.09 & $0.77,1.55$ & 1.01 & $0.65,1.57$ \\
\hline
\end{tabular}

Data are presented as odds ratio with $95 \%$ confidence interval (after adjustment for age and gender).

MetS, metabolic syndrome defined by IDF; Ref., reference category. Abdominal obesity, WC $>=$ P90; Low HDL cholesterol, HDL-C $<1.03$ $\mathrm{mmol} / \mathrm{L}$; Elevated FG, FG $>=5.6 \mathrm{mmol} / \mathrm{L}$; High triglyceride, triglyceride $(\mathrm{TG})>=1.7 \mathrm{mmol} / \mathrm{L}$; High blood pressure, $\mathrm{SBP}>=130 \mathrm{mmHg}$ or $\mathrm{DBP}>=85 \mathrm{mmHg}$.

* $p<0.05$, assessed by multiple logistic regression.

one component, $7.5 \%$ had two components, $2.0 \%$ had three components, and $0.3 \%$ had four components. Only four participants $(0.0 \%)$ had all five components.

\section{Associations Between MetS Components and Related} Factors

By single factor analysis, age, sex, and paternal education were significantly associated with MetS. To 
further characterize the risk and protective factors for MetS and its components, maternal education level and birth weight were added to the multivariate logistic regression (Table 5). Hereby, the sample size fell to 11,696 in the logistic regression model after exclusion of participants without related variables recorded. In the subsample $(n=11,696)$, multivariate analysis demonstrated that neither birth weight nor parental education was significantly associated with MetS itself, after adjustment for age and sex. Regarding individual MetS component, high birth weight was positively correlated with abdominal obesity (odds ratio, 1.48) but negatively associated with elevated FG (odds ratio, 0.49). Besides, high parental education level (university) was found to be positively associated with abdominal obesity in children (odds ratio of 1.30 and 1.35 for paternal and maternal education level, respectively).

\section{Discussion}

The current study evaluated the prevalence of MetS and its components in children and adolescents aged 7-18 years using the pediatric definition proposed by the IDF. The overall MetS prevalence was $2.3 \%$, higher in males than in females and most prevalent among older subjects. Regarding the prevalence of MetS components, abdominal obesity was most common, followed by low HDL-C level. Furthermore, birth weight and parental education were not correlated with MetS itself, whereas HBW was positively correlated with abdominal obesity.

The MetS prevalence is comparable to previous studies in China and its neighboring countries whereas it is lower than the results obtained from some highincome countries. For example, previous studies in China reported a prevalence of $1.4 \%$ in 2013 among 22,071 children across 6 cities of China ${ }^{23)}$ and $1.8 \%$ in 2015 based on a meta-analysis ${ }^{24)}$. Furthermore, a study in Korea reported that $2.1 \%$ of 2,330 adolescents aged 10-19 years had MetS ${ }^{25)}$. Studies from many high-income countries generally showed higher MetS rates than those in China. For instance, MetS rate was reported to be $4.5 \%$ during 1999-2004 among American adolescents aged 12-17 years and $4.7 \%$ in 2009 among Canadian youth aged 6-18 years (all studies mentioned in this paragraph adopted the IDF definition) ${ }^{9,}{ }^{10}$ ). The prevalence difference between China and these high-income countries is consistent with the lower obesity prevalence among Chinese children ${ }^{26)}$.

Distribution of MetS in different sex, age groups, economic development areas, and geographic locations in this study is supported by previous studies.
Boys were at a greater risk of developing MetS in the present study, consistent with a number of studies conducted in and outside of China ${ }^{7,9,23,27)}$. As shown in Table 3, we believe that the significantly higher rate of high BP, low HDL-C level, and elevated FG in boys $(4.6 \%, 15.8 \%$, and $4.1 \%)$ than that in the girls $(2.7 \%$, $12.9 \%$, and $1.8 \%$ ) contributed to this gender disparity. In the present study, MetS prevalence generally increased with age, similar to a study from the USA ${ }^{\text {? }}$ and another meta-analysis in China ${ }^{28)}$. Regarding the economic development level, increased MetS risk was detected in more developed regions, supporting previous results in China ${ }^{27)}$. Irrational dietary habits, more access to junk food, unhealthy lifestyle, and lack of health consciousness may contribute to overall effect induced by economic development level. A higher MetS prevalence was found in subjects who lived in the north, as reported in a previous study in China ${ }^{29)}$. Higher BP and obesity have also been detected more frequently among children from North China ${ }^{30,31)}$ than their counterparts in the south, contributing to the higher MetS prevalence in the north. Previous studies did show that people in North China are likely to take in more salt than people in South China ${ }^{32,33)}$, and they tend to spend more time indoors and perform less physical activity during the extremely cold winter days in the north, resulting in higher rate of elevated BP, obesity, and MetS ${ }^{34)}$.

Comparing the five components of MetS, abdominal obesity was the most prevalent component in this population, followed by low HDL-C level, which was in line with a previous study conducted among 8,764 Chinese adolescents (2012) ${ }^{23)}$. In addition, the prevalence of high BP (3.7\%) in our study was lower than prior data in China ${ }^{23)}$, probably caused by the relatively stricter high BP criteria in IDF definition than that in the Chinese BP definition ${ }^{35)}$.

Well-educated mothers are believed to be more health-conscious with more knowledge about obesity and MetS, leading to lower risk of developing MetS for both themselves and their children ${ }^{17)}$. However, in our study, we did not find any associations between parental education and MetS itself. It is possible that people's education level might not be perfectly consistent with their health consciousness and literacy because China is one of the fastest growing developing countries in the world. Still, we believe the association between parental education and MetS might exist in certain developed areas of China. Regarding the five MetS components, both HBW and high parental education were positively correlated with abdominal obesity in this study. It is easy to understand that children with HBW are at an increased risk of developing abdominal obesity because previous studies have 
already reported similar results ${ }^{36,37)}$. The interesting positive association between high parental education and abdominal obesity is worth noting. Adverse changes in lifestyle related to high parental education (e.g., more convenience foods, sedentary lifestyle, and more total energy per day) along with the inadequate health consciousness might increase the obesity risk for their children, overriding any positive effect of higher parent educational level. Results on the correlation between HBW and elevated FG are not consistent yet ${ }^{16)}$.

\section{Limitations}

More detailed information about parental employment, dietary intakes, physical activity, and other factors should be obtained in further studies, in order to analyze the association between these factors and MetS more accurately and to identify the risk and protective factors for MetS and its components. Another limitation was the cross-sectional nature of our observational study, preventing us from concluding anything regarding cause-effect relationships. A longitudinal, prospective study design would have helped to investigate the direct links among various MetS factors.

\section{Conclusion}

In conclusion, MetS itself was not common in Chinese children and adolescents, whereas it's certain components, particularly abdominal obesity and low HDL-C level, were far more prevalent. Children from North China and more developed areas and those at an older age were more likely to develop MetS. Further, HBW was positively associated with abdominal obesity. Strategies designed to prevent pediatric MetS in China should focus more on certain prevalent components, and the geographic and economic predilections of MetS should also be considered while making policies.

\section{Acknowledgments}

We gratefully acknowledge the participants for their continuous and enthusiastic participation in the investigation. Also, we appreciate doctors and nurses involved in this study for their clinical technical support.

\section{Notice of Grant Support}

This project has been funded by Special Research Grant for Non-profit Public Service of the Ministry of
Health of China (Grant No. 201202010), Guangdong Provincial Natural Science Foundation (Grant No. 2015A030313175 and Grant No. 2017A030313844); the Fundamental Research Funds for the Central Universities in SYSU (Grant No. 15ykpy09), and the Sanming Project of Medicine in Shenzhen (Grant No. SZSM201803061). The funders had no role in study design, data collection and analysis, decision to publish, or preparation of the manuscript.

\section{Conflicts of Interest Statement}

None.

\section{Authorship}

Yanna Zhu, Jun Ma, and Yajun Chen designed the research study. Zhiyong Zou, Yinghua Ma, Haijun Wang, Jiayou Luo, Xin Zhang, Chunyan Luo, Hong Wang, Haiping Zhao, and Dehong Pan carried out the experiments and collected the data. Hao Zheng, Jin Jing, Per Sangild, and Biraj M Karmacharya analyzed the data. All authors were involved in writing the paper and had final approval of the submitted and published versions.

\section{References}

1) Alberti KG, Zimmet P, Shaw J: Metabolic syndrome--a new world-wide definition. A Consensus Statement from the International Diabetes Federation. Diabetic medicine, 2006; 23: 469-480

2) Povel CM, Beulens JW, van der Schouw YT, Dolle ME, Spijkerman AM, Verschuren WM, Feskens EJ, Boer JM: Metabolic syndrome model definitions predicting type 2 diabetes and cardiovascular disease. Diabetes care, 2013 ; 36: 362-368

3) Glueck CJ, Woo JG, Khoury PR, Morrison JA, Daniels SR, Wang P: Adolescent oligomenorrhea (age 14-19) tracks into the third decade of life (age 20-28) and predicts increased cardiovascular risk factors and metabolic syndrome. Metabolism, clinical and experimental, 2015; 64: 539-553

4) Cook S, Weitzman M, Auinger P, Nguyen M, Dietz WH: Prevalence of a metabolic syndrome phenotype in adolescents: findings from the third National Health and Nutrition Examination Survey, 1988-1994. Archives of pediatrics $\&$ adolescent medicine, 2003; 157: 821-827

5) de Ferranti SD, Gauvreau K, Ludwig DS, Neufeld EJ, Newburger JW, Rifai N: Prevalence of the metabolic syndrome in American adolescents: findings from the Third National Health and Nutrition Examination Survey. Circulation, 2004; 110: 2494-2497

6) Zimmet P, Alberti KG, Kaufman F, Tajima N, Silink M, Arslanian S, Wong G, Bennett P, Shaw J, Caprio S et al: The metabolic syndrome in children and adolescents - an IDF consensus report. Pediatric diabetes, 2007; 8: 299- 
306

7) Aboul Ella NA, Shehab DI, Ismail MA, Maksoud AA: Prevalence of metabolic syndrome and insulin resistance among Egyptian adolescents 10 to 18 years of age. Journal of clinical lipidology, 2010; 4: 185-195

8) Duncan GE, Li SM, Zhou XH: Prevalence and trends of a metabolic syndrome phenotype among u.s. Adolescents, 1999-2000. Diabetes care, 2004; 27: 2438-2443

9) Ford ES, Li C, Zhao G, Pearson WS, Mokdad AH: Prevalence of the metabolic syndrome among U.S. adolescents using the definition from the International Diabetes Federation. Diabetes care, 2008; 31: 587-589

10) MacPherson M, de Groh M, Loukine L, Prud'homme D, Dubois L: Prevalence of metabolic syndrome and its risk factors in Canadian children and adolescents: Canadian Health Measures Survey Cycle 1 (2007-2009) and Cycle 2 (2009-2011). Health promotion and chronic disease prevention in Canada: research, policy and practice, 2016; 36: 32-40

11) Hossain P, Kawar B, El Nahas M: Obesity and diabetes in the developing world--a growing challenge. The New England journal of medicine, 2007; 356: 213-215

12) Stevens J, Truesdale KP, Katz EG, Cai J: Impact of body mass index on incident hypertension and diabetes in Chinese Asians, American Whites, and American Blacks: the People's Republic of China Study and the Atherosclerosis Risk in Communities Study. American journal of epidemiology, 2008; 167: 1365-1374

13) Deurenberg P, Deurenberg-Yap M, Guricci S: Asians are different from Caucasians and from each other in their body mass index/body fat per cent relationship. Obesity reviews: an official journal of the International Association for the Study of Obesity, 2002; 3: 141-146

14) Ma GS, Ji CY, Ma J, Mi J, Yt Sung R, Xiong F, Yan WL, Hu XQ, Li YP, Du SM et al: Waist circumference reference values for screening cardiovascular risk factors in Chinese children and adolescents. Biomedical and environmental sciences, 2010; 2321-2331

15) Baxi R, Vasan SK, Hansdak S, Samuel P, Jeyaseelan V, Geethanjali FS, Murray RR, Venkatesan P, Thomas N: Parental determinants of metabolic syndrome among adolescent Asian Indians: A cross-sectional analysis of parentoffspring trios. Journal of diabetes, 2016; 8: 494-501

16) Xiao X, Zhang ZX, Li WH, Feng K, Sun Q, Cohen HJ, Xu T, Wang H, Liu AM, Gong XM et al: Low birth weight is associated with components of the metabolic syndrome. Metabolism: clinical and experimental, 2010; 59: 1282-1286

17) Choi BY, Lee DC, Chun EH, Lee JY: The Relationship between Metabolic Syndrome and Childhood Maternal Education Level, Job Status Findings from the Korean National Health and Nutrition Examination, 2007-2009. Korean journal of family medicine, 2014; 35: 207-215

18) Chen Y, Ma L, Ma Y, Wang H, Luo J, Zhang X, Luo C, Wang $\mathrm{H}$, Zhao $\mathrm{H}$, Pan D et al: A national school-based health lifestyles interventions among Chinese children and adolescents against obesity: rationale, design and methodology of a randomized controlled trial in China. BMC public health, 2015; 15: 210

19) F Wang QG, S Wang, Q Li, PD Jones: A new estimation of urbanization's contribution to the warming trend in
China. Journal of Climate, 2015; 28: 8923

20) China Statistical Yearbook: National Bureau of Statistics of China (2016). http: //www.stats.gov.cn/tjsj/ndsj/2016/ indexch.htm. Accessed 16 April 2019

21) International statistics classification of diseases and related health problems (WHO 10th revision, Chapter XVI). (2016) World Health Organization. http: //apps.who.int/ classifications/icd10/browse/2016/en\#/P07.0. Accessed 16 April 2019

22) World Health Organization. (2007) WHO growth reference for children aged between 5-19 years. Available online: https://www.who.int/growthref/who2007_bmi_ for_age/en/. Accessed 26th August 2019.

23) Chinese Work Group of Pediatric Metabolic Syndrome: Prevalence of metabolic syndrome of children and adolescent students in Chinese six cities. Zhonghua Er Ke Za Zhi, 2013; 51: 409-413

24) Ye P, Yan Y, Ding W, Dong H, Liu Q, Huang G, Mi J: Prevalence of metabolic syndrome in Chinese children and adolescents: a Meta-analysis. Zhonghua Liu Xing Bing Xue Za Zhi, 2015; 36: 884-888

25) Kim S, So WY: Prevalence of Metabolic Syndrome among Korean Adolescents According to the National Cholesterol Education Program, Adult Treatment Panel III and International Diabetes Federation. Nutrients, 2016; 8: pii: E588

26) Song Y, Wang HJ, Dong B, Ma J, Wang Z, Agardh A: 25 -year trends in gender disparity for obesity and overweight by using WHO and IOTF definitions among Chinese school-aged children: a multiple cross-sectional study. BMJ open, 2016; 6: e011904

27) Wang J, Zhu Y, Cai L, Jing J, Chen Y, Mai J, Ma L, Ma Y, Ma J: Metabolic syndrome and its associated early-life factors in children and adolescents: a cross-sectional study in Guangzhou, China. Public health nutrition, 2016; 19: 1147-1154

28) Li R, Li W, Lun Z, Zhang H, Sun Z, Kanu JS, Qiu S, Cheng Y, Liu Y: Prevalence of metabolic syndrome in Mainland China: a meta-analysis of published studies. BMC public health, 2016; 16: 296

29) Li P, Jiang R, Li L, Liu C, Yang F, Qiu Y: Prevalence and risk factors of metabolic syndrome in school adolescents of northeast China. J Pediatr Endocrinol Metab, 2014; 27: 525-532

30) Xi B, Li H, Li S, Mi J: Recent prevalence of hypertension among Chinese children and adolescents based on 2010 China national blood pressure references. International journal of cardiology, 2014; 174: 870-871

31) Zhou Y, Wang S, Zhang Q, Zhang Y, Wang T: Prevalence of overweight and obesity among children and adolescents increased rapidly in Chinese rural regions while level off in urban areas. International journal of cardiology, 2016; 223: 61-62

32) Zhang JG, Wang ZH, Du WW, Su C, Jiang HR, Huang FF, Jia XF, Ouyang YF, Li L, Wang Y: Dietary sodium intake of adult residents in 15 provinces of China in 2015. Chinese journal of preventive medicine, 2019; 53: 455-458

33) Zhao L, Stamler J, Yan LL, Zhou B, Wu Y, Liu K, Daviglus ML, Dennis $\mathrm{BH}$, Elliott $\mathrm{P}$, Ueshima $\mathrm{H}$ et al: Blood pressure differences between northern and southern Chi- 
nese: role of dietary factors: the International Study on Macronutrients and Blood Pressure. Hypertension, 2004; 43: $1332-1337$

34) Zhang D, Liu X, Liu Y, Sun X, Wang B, Ren Y, Zhao Y, Zhou J, Han C, Yin L: Leisure-time physical activity and incident metabolic syndrome: a systematic review and dose-response meta-analysis of cohort studies. Metabolism: clinical and experimental, 2017; 75: 36-44

35) J. Mi TYW, L.H. Meng: Development of blood pressure reference standards for Chinese children and adolescents. Chin J Evid Based Pediatr, 2010; 5: 4-14
36) Ansari H, Qorbani M, Rezaei F, Djalalinia S, Asadi M, Miranzadeh S, Motlagh ME, Bayat S, Safiri S, Safari O: Association of birth weight with abdominal obesity and weight disorders in children and adolescents: the weight disorder survey of the CASPIAN-IV Study. Journal of cardiovascular and thoracic research, 2017; 9: 140-146

37) Suder A, Chrzanowska M: Risk factors for abdominal obesity in children and adolescents from Cracow, Poland (1983-2000). Journal of biosocial science, 2015; 47: 203219 